\title{
P03-7-29 Poster session
}

\section{Development and characterisation of a triple-negative breast cancer multicellular spheroidal model}

\author{
Keith N Ncube ${ }^{1}$, Vanessa Steenkamp ${ }^{1}$, Duncan A Cromarty ${ }^{1}$, Iman Van Den Bout ${ }^{2}$, Werner Cordier ${ }^{1}$ \\ ${ }^{1}$ Department of Pharmacology, University of Pretoria, South Africa, ${ }^{2}$ Centre for Neuroendocrinology, University of \\ Pretoria, South Africa
}

\section{Background}

In vitro monolayer (2D) cell culture models have limited predictive ability for the clinical outcome of potential anti-cancer therapies as these models fail to reproduce the physiological cues of the tumour micromilieu. Three-dimensional (3D) multicellular spheroids are used as an alternative, as they better resemble the in vivo tumour environment with regards to cellular interactions and chemical gradients. Triple-negative breast cancer (TNBC) is a sub-type of breast cancer associated with resistance, relapse and lack of target specificity. This study sought to develop and characterise a spheroidal model of TNBC.

\section{Methods}

To select a reproducible TNBC spheroidal model, BT-20 and MDA-MB 231 cell lines were compared and grown using the liquid overlay and hanging-drop assays. Growth was assessed using phase contrast microscopy and the bicinchoninic acid protein content analysis assay. Viability was assessed using the fluorescein diacetate (FDA)/propidium iodide (PI) assay. Haematoxylin and eosin staining was used for morphological evaluation. Susceptibility to doxorubicin was assessed in 2D-monolayer cultures using sulphorhodamine B staining, FDA/PI-staining, and acid phosphatase conversion. The effect of toxic concentrations of doxorubicin on spheroids' morphology and enzymatic activity was assessed and compared to 2D-monolayer cultures.

\section{Results}

Only BT-20 cells cultured using the liquid overlay assay formed reproducible dense spheroids which were subsequently used for characterisation. Spheroid diameter decreased from day four $(949 \mu \mathrm{m})$ to day ten $(787 \mu \mathrm{m})$, while protein content increased slightly in parallel ( 7.0 to $8.5 \mu \mathrm{g} / \mathrm{spheroid),} \mathrm{suggesting} \mathrm{spheroidal} \mathrm{compaction} \mathrm{rather} \mathrm{than} \mathrm{cell} \mathrm{death.}$ The outer spheroid region had viable, well-nourished cells, while smaller, membrane-compromised cells were localized in the inner spheroid region with a clear distinction between the layers. $\mathrm{The} \mathrm{IC}_{25}(130 \mathrm{nM}), \mathrm{IC}_{50}(320 \mathrm{nM})$ and $\mathrm{IC}_{75}(1580$ $\mathrm{nM}$ ) of doxorubicin induced a dose-dependent reduction of monolayer cell viability and acid phosphatase activity. However, these concentrations did not alter spheroid size and acid phosphatase activity, suggesting resistance incurred by its 3D-conformation.

\section{Conclusion}

Heterogeneous cellular architecture and resistance of BT-20 spheroids resemble in vivo attributes of TNBC. It is essential to incorporate such a 3D model in preclinical drug screens in order to facilitate translational cancer research and potentially discover optimally effective drugs against TNBC. 